\title{
Pore-scale simulations of rarefied gas flows in ultra-tight porous media
}

\author{
Minh Tuan $\mathrm{Ho}^{\mathrm{a}}$, Lianhua Zhu ${ }^{\mathrm{a}, \mathrm{b}}$, Lei Wu ${ }^{\mathrm{a}}$, Peng Wang ${ }^{\mathrm{a}}$, Zhaoli Guo ${ }^{\mathrm{b}}$, Jingsheng $\mathrm{Ma}^{\mathrm{c}}$, Yonghao \\ Zhang ${ }^{a, *}$ \\ ${ }^{a}$ James Weir Fluids Laboratory, Department of Mechanical and Aerospace Engineering, University of Strathclyde, \\ Glasgow G1 1XJ, UK \\ ${ }^{b}$ State Key Laboratory of Coal Combustion, School of Energy and Power, Huazhong University of Science and \\ Technology, Wuhan, 430074, China \\ ${ }^{c}$ Institute of Petroleum Engineering, School of Energy, Geoscience, Infrastructure and Society, Heriot-Watt \\ University, Edinburgh EH14 4AS, UK
}

\begin{abstract}
An in-depth understanding of gas transport in ultra-tight porous media is the key to quantifying flow properties of shale rocks with pore space as small as a few nanometers, where the gas rarefaction effects play a major role. As the conventional fluid mechanics theory fails to describe non-equilibrium rarefied flow, we resort to the gas kinetic theory and directly simulate gas flow inside the porous media utilising the digital images of porous media where the pore space is resolved. The Boltzmann model equation is solved by the discrete velocity method (DVM), which can accurately predict the permeability enhancement caused by rarefaction effects. Our simulations for different porous media show that the commonly-used standard lattice Boltzmann method (LBM) cannot describe rarefaction effects, although the kinetic boundary condition, which helps to capture velocity-slip, can extend the validity of the LBM to the slip flow regime. The heuristic Klinkenberg-type models proposed for all the flow regimes often involve many unknown empirical parameters, which may be calibrated by our simulations. However, these parameters are different for each porous medium and also depend on flow conditions, so these models are not of any prac-
\end{abstract}


tical use. By contrast, our kinetic solver can accurately predict apparent permeability without introducing any empirical parameters, which lays firm foundation for upscaling. As the large flow paths with least flow resistance dominate the overall permeability, the requirement on the velocityspace resolution is significantly reduced for our DVM simulations to predict accurate permeability with affordable computational costs, which offers a promising new way for digital rock analysis.

Keywords: rarefied gas dynamics, tight porous media, permeability correction, shale gas

\section{Introduction}

Although the unconventional gases accounted for $40 \%$ of the recoverable resources of natural gas [1], their production contributed only $14 \%$ of nature gas supply in 2010 . The share of un4 conventional gases in the nature gas provision is expected to rise to $21 \%$ and $32 \%$ in 2020 and 5 2035, respectively [2]. However, the production of unconventional gases poses an unprecedented challenge to reservoir engineers, as gas transport in unconventional reservoirs, such as shale rocks, is poorly understood [3, 4]. Owning to extremely low permeability and complex flow paths with micro/nano-scale pore space, the conventional fluid dynamics theory fails to capture important rarefaction effects.

When gas molecules collide frequently with pore surface in comparison with the collisions among themselves, gas rarefaction effects become significant thus influence gas transport in the porous media. The rarefaction level can be indicated by the Knudsen number $K n$, defined as the

\footnotetext{
${ }^{*}$ Corresponding author

Email address: yonghao.zhang@strath.ac.uk (Yonghao Zhang)
} 
ratio of the molecular mean free path $\lambda$ to the characteristic flow length $L$, i.e.

$$
K n=\frac{\lambda}{L}
$$

14 where $\lambda=\frac{\mu\left(\hat{T}_{0}\right)}{\bar{p}} \sqrt{\frac{\pi R \hat{T}_{0}}{2}}$, and $R, \bar{p}$ and $\mu\left(\hat{T}_{0}\right)$ are the specific gas constant, mean gas pressure and dynamic viscosity at a reference temperature $\hat{T}_{0}$, respectively. According to $K n$, gas flows can

be roughly categorized into four regimes: continuum $(K n<0.001)$, slip $(0.001<K n<0.1)$, transition $(0.1<K n<10)$ and free-molecular $(K n>10)$ flow regimes. The Navier-Stokes equations are only applicable in the continuum flow regime and their validity might be extended to the slip flow regime by introducing velocity-slip boundary condition at the solid surfaces [5, 6]. In the transition and free molecular flow regimes, the linear constitutive relations as assumed in the Navier-Stokes equations are no longer valid [7].

To predict the gas permeability through tight porous media where the Knudsen number is non-negligible, rarefaction effects which enhance permeability should be taken into account. Indeed, the Darcy's law was first extended to the slip regime $(K n<0.1)$, i.e. the Klinkenberg model, by considering gas velocity-slip at surface [8]. Recently, more models have been proposed to further extend the Klinkenberg slip-flow correction to all the flow regimes by implementing the second-order velocity-slip boundary condition or the so-called Knudsen diffusion mechanism [9-12]. However, these flow models include many empirical parameters that are difficult to be determined experimentally, theoretically or numerically.

The gas kinetic theory approach is indispensable to describe gas flow in all the flow regimes. As direct simulation Monte Carlo (DSMC) method is extremely expensive for simulating lowspeed flows in tight porous media [13], the kinetic model equations such as Bhatnagar-Gross- 
Krook (BGK) [14], ellipsoidal-statistical BGK (ES-BGK) [15] or Shakhov (S) [16] model, can be solved to provide accurate results to unravel gas transport mechanisms at the pore scale. Among various numerical methods for solving the Boltzmann model equations, lattice Boltzmann model (LBM) [17] is well developed and extensively used for modelling flows in porous media thanks to the ease of boundary implementation on complex surfaces [18-22]. Although the conventional LBM can be derived from the BGK equation, it fails to capture rarefaction effects in simple flows due to limited number of discrete velocities [23, 24]. It is demonstrated that considerably highorder LBMs with more discrete velocities are needed to capture the Knudsen paradox phenomena in a straight channel [25]. Therefore, accuracy of the conventional LBM for porous media flows in the transition and free-molecular regimes is still questionable, and higher-order LBMs, which are a special form of discrete velocity method (DVM) [26, 27], are required. All flow regimes in capillaries of various cross sections have been accurately predicted by DVM [28--32].

Here, we will directly solving the BGK kinetic equation using DVM and LBM for rarefied gas flows through porous media at the pore scale where the detailed flow paths are obtained from finely-resolved digital images. So we can predict gas permeability directly to avoid many unknown empirical parameters. The results will show how rarefaction affects the apparent permeability and provide vital information for reservoir simulations.

The remainder of this paper is structured as follows. The governing equation and numerical methods are described in Section 2, Section 3 briefly introduces the heuristic models on permeability correction that are commonly used in literature. The numerical results are presented and compared with the heuristic models in Section 4 . Finally, the conclusions are drawn in Section 5 


\section{Kinetic model and numerical methods}

The Boltzmann equation, which is the cornerstone of rarefied gas dynamics, describes the temporal $\hat{t}$ and spatial $\hat{\mathbf{x}}$ evolution of the distribution function $\hat{f}(\hat{\mathbf{x}}, \hat{\mathbf{v}}, \hat{t})$ of gas molecules with the velocity $\hat{\mathbf{v}}$, i.e.

$$
\frac{\partial \hat{f}}{\partial \hat{t}}+\hat{\mathbf{v}} \cdot \frac{\partial \hat{f}}{\partial \hat{\mathbf{x}}}=I\left(\hat{f}, \hat{f}_{*}\right)
$$

where the Boltzmann collision integral $I\left(\hat{f}, \hat{f}_{*}\right)$ represents the rate at which the distribution function varies before collision $\hat{f}$ and after collision $\left(\hat{f}_{*}\right)$. Because of the complex five-fold integral $I$ and multidimensional phase space (3D in the physical domain, 3D in the velocity domain and additional 1D in temporal domain for unsteady flows), numerical solution of this nonlinear integro-differential equation for any realistic flow problems is difficult and tremendously expensive in terms of computational costs. Therefore, the collision integral $I$ is usually simplified by a relaxation-time collision model for the most engineering applications.

The linearised BGK equation is one of such simplified Boltzmann model equations, which can be employed to describe low-speed gas flows in tight porous media. The distribution function is linearised in the standard manner as $\hat{f}=\hat{f_{e q}}(1+h)$ [33], where the Maxwellian distribution function $\hat{f_{e q}}$ is given below,

$$
\hat{f}_{e q}=\frac{\hat{n}_{e q}}{\left(2 \pi R \hat{T}_{0}\right)^{3 / 2}} \exp \left(-\frac{|\hat{\mathbf{v}}|^{2}}{2 R \hat{T}_{0}}\right),
$$

where the global equilibrium number density $\hat{n}_{e q}$ is related to the mean gas pressure by the ideal gas law $\hat{n}_{e q}=\bar{p} / m R \hat{T}_{0}$, in which $m$ is the molecular mass. Therefore, the (dimensionless) perturbed distribution function $h(\mathbf{x}, \mathbf{v})$ is governed by the linearised BGK equation as

$$
\frac{\partial h}{\partial t}+\mathbf{v} \cdot \frac{\partial h}{\partial \mathbf{x}}=-\frac{1}{\tau}\left(h-h^{e q}\right),
$$


where the perturbed equilibrium distribution function $h^{e q}$ is determined by

$$
h^{e q}=\varrho+2 \mathbf{u} \cdot \mathbf{v}+\mathcal{T}\left(|\mathbf{v}|^{2}-\frac{3}{2}\right)
$$

73 and the dimensionless relaxation time is defined as

$$
\tau=\frac{\mu\left(\hat{T}_{0}\right) / \bar{p}}{L / v_{m}}=\frac{2 K n}{\sqrt{\pi}}
$$

Eq. (8) can be transformed into (representing use $\tilde{h}_{k}$ instead of $h_{k}$ )

$$
\tilde{h}_{k}\left(\mathbf{x}+\mathbf{v}^{(k)} \Delta t, t+\Delta t\right)-\tilde{h}_{k}(\mathbf{x}, t)=-\frac{2 \Delta t}{2 \tau+\Delta t}\left[\tilde{h}_{k}(\mathbf{x}, t)-h_{k}^{e q}(\mathbf{x}, t)\right] .
$$

where $v_{m}=\sqrt{2 R \hat{T}_{0}}$ is the most probable speed.

\subsection{Lattice Boltzmann method}

The standard LBM can be considered as a specific finite difference scheme on uniform grid of the BGK equation (4) [34]. If we project the continuum molecular velocity into a specific set of discretised velocity $\mathbf{v}^{(k)}(k=1,2, . ., N)$, and then integrate discrete version of Eq. (4) along the characteristic line, i.e. direction of the discrete velocity, we obtain

Define $\tilde{h}_{k}=h_{k}+\frac{\Delta t}{2 \tau}\left[h_{k}-h_{k}^{e q}\right]$, then we have

$$
h_{k}=\frac{2 \tau}{2 \tau+\Delta t} \tilde{h}_{k}+\frac{\Delta t}{2 \tau+\Delta t} h_{k}^{e q} .
$$

$$
h_{k}\left(\mathbf{x}+\mathbf{v}^{(k)} \Delta t, t+\Delta t\right)-h_{k}(\mathbf{x}, t)=-\frac{\Delta t}{2 \tau}\left[h_{k}\left(\mathbf{x}+\mathbf{v}^{(k)} \Delta t, t+\Delta t\right)-h_{k}^{e q}\left(\mathbf{x}+\mathbf{v}^{(k)} \Delta t, t+\Delta t\right)+h_{k}(\mathbf{x}, t)-h_{k}^{e q}(\mathbf{x}, t)\right] .
$$


Define the effective relaxation time $\tilde{\tau}=\frac{1}{2}+\frac{\tau}{\Delta t}$, then

$$
\tilde{h}_{k}\left(\mathbf{x}+\mathbf{v}^{(k)} \Delta t, t+\Delta t\right)-\tilde{h}_{k}(\mathbf{x}, t)=-\frac{1}{\tilde{\tau}}\left[\tilde{h}_{k}(\mathbf{x}, t)-h_{k}^{e q}(\mathbf{x}, t)\right] .
$$

85

One major feature of the standard LBM is on-lattice, i.e. the discretised molecular speed $\left|\mathbf{v}^{(k)}\right|$ is chosen to be equal to the grid size in the corresponding direction if $\Delta t=1$. The lattice models of D2Q9 and D3Q19 are used for 2D and 3D simulations, respectively, in this study.

\subsection{Discrete velocity method}

DVM is one of the most common deterministic approaches to solve the Boltzmann equation and its simplified models [26, 27], which projects the continuous molecular velocity space $\mathbf{v}$ into a set of fixed $N_{v}$-discrete velocities $\mathbf{v}^{(k)}\left(k=1,2, . ., N_{v}\right)$. Consequently, the BGK equation (4) is replaced by a system of $N_{v}$-independent equations. Since only the steady-state solution is of interest, the time-derivative term in Eq. (4) can be omitted in the DVM simulation, i.e.

$$
\mathbf{v}^{(k)} \cdot \frac{\partial h_{k}}{\partial \mathbf{x}}=-\frac{1}{\tau}\left(h_{k}-h_{k}^{e q}\right)
$$

The upwind schemes are used to approximate the spatial derivatives of the advection term, e.g. the gradient component of $h$ at the fluid point $\mathbf{x}$ projected in the $x_{j}$ coordinate axis $(j=1,2,3)$ is evaluated as follows

$\frac{\partial h(\mathbf{x}, \mathbf{v})}{\partial x_{j}} \approx \mathcal{D}(h(\mathbf{x}, \mathbf{v}))_{j}=\operatorname{sgn}\left(v_{j}\right)\left[C_{0} h(\mathbf{x}, \mathbf{v})+C_{1} h\left(\mathbf{x}-\operatorname{sgn}\left(v_{j}\right) \Delta x \mathbf{i}_{j}, \mathbf{v}\right)+C_{2} h\left(\mathbf{x}-\operatorname{sgn}\left(v_{j}\right) 2 \Delta x \mathbf{i}_{j}, \mathbf{v}\right)\right]$,

where $\operatorname{sgn}, \Delta x$ and $\mathbf{i}_{j}$ are the sign (signum) function, the constant grid size, and the unit vector of the $x_{j}$ coordinate axis, respectively. The constants $\left(C_{0}, C_{1}, C_{2}\right)$ are equal to $(1.5,-2,0.5) / \Delta x$ for the second-order-accurate scheme and $(1,-1,0) / \Delta x$ for the first-order-accurate scheme. In this 
study, the second-order-accurate scheme is used by default while the first-order-accurate scheme is automatically deployed if the previous grid point in the $v_{j}$-direction is located on the solid surface or the outer boundary faces. The Cartesian velocity grid generated by half-range GaussHermit quadrature is employed in this study [35]. In the forthcoming sections, we use $D 2 Q N_{v}$ (or $D 3 Q N_{v}$ ) to denote the DVM simulations using the discrete velocity set of $N_{v}$ points in a 2D (or 3D) problem.

\subsection{Boundary conditions}

Boundary conditions for the BGK equation should be specified at the pore surfaces and the outer faces of a porous medium. Gas-surface interaction is modeled by the Maxwell diffusespecular reflection, i.e.

$$
h(\mathbf{v} \mid \mathbf{v} \cdot \mathbf{n}>0)=\alpha_{t} \varrho_{s}(\mathbf{n})+\left(1-\alpha_{t}\right) h(\mathbf{v}-2 \mathbf{n}(\mathbf{v} \cdot \mathbf{n})),
$$

where $\mathbf{n}, \varrho_{s}, \alpha_{t}$ are the normal unit vector of the solid surface, the perturbed gas number density on the solid surface, and the tangential momentum accommodation coefficient (TMAC), respectively. TMAC represents the diffuse portion of the reflected molecules, e.g. fully diffuse or fully specular reflection corresponds to $\alpha_{t}=1$ or $\alpha_{t}=0$ respectively. This study uses the diffuse boundary condition $\alpha_{t}=1$ at the solid surfaces for both the DVM and LBM simulations [36, 37]. The perturbed gas number density on the solid surface is computed from the non-penetration condition, i.e. zero-mass flux through the solid surface

$$
\varrho_{S}(\mathbf{n})=-\frac{\int_{\mathbf{v} \cdot \mathbf{n}<0} \mathbf{v} \cdot \mathbf{n} \exp \left(-|\mathbf{v}|^{2}\right) h \mathrm{~d} \mathbf{v}}{\int_{\mathbf{v} \cdot \mathbf{n}>0} \mathbf{v} \cdot \mathbf{n} \exp \left(-|\mathbf{v}|^{2}\right) \mathrm{d} \mathbf{v}} .
$$

A porous medium can be constructed by the periodic replica of the representative elementary volume, e.g. a rectangular cuboid with the edge length, width and height of $L_{1}, L_{2}$, and $L_{3}$ in 


$$
\begin{aligned}
& h\left(x_{1}, x_{2}^{\text {min }}, x_{3}, v_{1}, v_{2}, v_{3}\right)=h\left(x_{1}, x_{2}^{\text {min }}, x_{3}, v_{1},-v_{2}, v_{3}\right), \quad \text { when } v_{2}>0, \\
& h\left(x_{1}, x_{2}^{\text {max }}, x_{3}, v_{1}, v_{2}, v_{3}\right)=h\left(x_{1}, x_{2}^{\text {max }}, x_{3}, v_{1},-v_{2}, v_{3}\right), \quad \text { when } v_{2}<0, \\
& h\left(x_{1}, x_{2}, x_{3}^{\text {min }}, v_{1}, v_{2}, v_{3}\right)=h\left(x_{1}, x_{2}, x_{3}^{\text {min }}, v_{1}, v_{2},-v_{3}\right), \quad \text { when } v_{3}>0, \\
& h\left(x_{1}, x_{2}, x_{3}^{\text {max }}, v_{1}, v_{2}, v_{3}\right)=h\left(x_{1}, x_{2}, x_{3}^{\text {max }}, v_{1}, v_{2},-v_{3}\right), \quad \text { when } v_{3}<0 .
\end{aligned}
$$

At the lateral outer faces of the porous medium, the plane-symmetric boundary conditions are implemented by the specular reflection

\subsection{Macroscopic variables}

The perturbed number density $\varrho$, velocity $\mathbf{u}$ and temperature $\mathcal{T}$ are calculated as the moments of the perturbed distribution function $h$ (or $\tilde{h}$ ) over the velocity space, i.e.

$$
\varrho=\int f_{e q} h \mathrm{~d} \mathbf{v}, \quad \mathbf{u}=\int \mathbf{v} f_{e q} h \mathrm{~d} \mathbf{v}, \quad \mathcal{T}=\frac{2}{3} \int|\mathbf{v}|^{2} f_{e q} h \mathrm{~d} \mathbf{v}-\varrho,
$$

where the dimensionless equilibrium distribution function is given as

$$
f_{e q}=\pi^{-3 / 2} \exp \left(-|\mathbf{v}|^{2}\right) .
$$


The dimensionless apparent permeability $k$, which is scaled by $L^{2}$, is calculated as

$$
k=\frac{K n}{\sqrt{\pi}} G_{p}
$$

The dimensionless mass flow rate $G_{p}$, which is normalized by $L_{2} L_{3} \Delta \hat{p} / v_{m}$, can be calculated from the velocity flow field

$$
G_{p}=2 \int_{x_{3}^{\min }}^{x_{3}^{\max }} \int_{x_{2}^{\min }}^{x_{2}^{\max }} u_{1}\left(x_{2}, x_{3}\right) \mathrm{d} x_{2} \mathrm{~d} x_{3},
$$

where $\Delta \hat{p}$ is the total pressure drop. The apparent permeability $k$ is calculated every 1000 iterations until the following convergence criterion

$$
\left|\frac{k^{(l)}-k^{(l-1000)}}{k^{(l)}}\right|<10^{-6},
$$

is satisfied, where $l$ is the number of iterations.

In order to appropriately compare the pore-scale rarefaction effects in different samples of various sizes, the effective Knudsen number $K n_{l}$ is defined as

$$
K n_{l}=\frac{\lambda}{L^{*}}=\frac{K n}{L^{*} / L}
$$

where the average pore size $L^{*}$ is defined as

$$
L^{*} / L=\sqrt{\frac{12 k_{\infty}}{\epsilon}} \text { for } 2 \mathrm{D}, \quad L^{*} / L=\sqrt{\frac{8 k_{\infty}}{\epsilon}} \text { for 3D. }
$$

With this definition, the average pore size $L^{*}$ is equal to channel height (or tube radius) in the case of ideal 2D (or 3D) porous medium that consists of uniform straight channels (or straight tubes). In this study, the intrinsic (liquid) permeability $k_{\infty}$, which depends only on the porous matrix, is obtained from the numerical data of $k$ at $K n \rightarrow 0$. The porosity $\epsilon$ of a porous model in Eq. (24) is determined by the ratio of the number of fluid points to the total number of points, i.e. the percentage of voids in the digital image of a porous medium sample. 


\section{Heuristic models on permeability correction}

The intrinsic permeability is a property of the porous medium which can be described by the Darcy's law. But for gas flows, rarefaction effects including velocity-slip and Knudsen diffusion will enhance the permeability. To consider the velocity-slip effect, a linear correction for gas permeability through an idealized porous medium composed of uniform tubes of radius $r$ was first introduced by Klinkenberg [8]

$$
\frac{k}{k_{\infty}}=1+\frac{4 c \lambda}{r}=1+4 c K n_{l},
$$

where the proportionality factor $c$ is commonly chosen as unity. The Klinkenberg's linear correction on the effective Knudsen number is usually employed in the following form $k / k_{\infty}=1+b_{k} / \bar{p}$, as it is more convenient to correlate the slippage factor $b_{k}$ with measurements. Calibration of the Klinkenberg's slippage factor for tight porous media is discussed in Ref. [12].

However, in ultra-tight porous media, Knudsen diffusion becomes important as the effective Knudsen number $K n_{l}$ can be substantial. Therefore, the Klinkerberg's linear correction is not sufficient. Following the empirical scaling laws proposed by Beskok and Karniadakis for straight pipe/tube/channel flows [38], Civan proposed a second-order correction in terms of $K n_{l}$ for tight porous media [10]

$$
\frac{k}{k_{\infty}}=C_{r}\left(1+\frac{4 K n_{l}}{1+K n_{l}}\right)
$$

where the term in the parentheses derived by correlating velocity slip model with numerical velocity profile. The rarefaction coefficient $C_{r}=\left(1+\alpha K n_{l}\right)$ accounts for dependence of viscosity on the level of rarefaction [38]. The empirical coefficient $\alpha$ is determined by correlating mass flow 
rate with numerical and experimental data as follows

$$
\alpha=\frac{1.358}{1+0.178 K n_{l}^{-0.4348}} .
$$

Here, this second-order correction is referred as the Beskok-Karniadakis-Civan (BKC) model. The tortuosity $\tau_{h}$ of hydraulic preferential flow paths in porous medium is taken into account by adjusting the average pore size in Eq. (24), i.e.

$$
L^{*} / L=\sqrt{\frac{8 \tau_{h} k_{\infty}}{\epsilon}} .
$$

In order to use the above 3D model for 2D porous media, $\alpha$ should be calibrated by channel flows rather than pipe flows. For a channel of length-to-height ratio of $20, \alpha$ is set to be 2.2. In addition, the factor of 4 in the last term of Eqs. [25), (26) is replaced by 6 for 2D porous media [38].

Darabi et al.[11] proposed the apparent permeability function (APF) to consider both the slip flow and Knudsen diffusion mechanisms for ultra-tight porous media as

$$
\frac{k}{k_{\infty}}=1+\left(\frac{2}{\alpha_{t}}-1\right) 4 K n_{l}+\frac{\epsilon}{\tau_{h}}\left(\delta^{\prime}\right)^{D_{f}-2} \frac{64}{3 \pi} K n_{l},
$$

where $\delta^{\prime}$ is the ratio of normalized molecular radius to average pore radius, $D_{f}$ is the fractal dimension of the pore surface. The slip term (the second term) in APF is very close to that of Klinkenberg model in the case of diffuse accommodation $\left(\alpha_{t}=1\right)$. This is also a linear correction in terms of the effective Knudsen number, the same as the Klinkenberg's model but with greater proportional factor owning to the additional Knudsen diffusion term (the third term).

\section{Numerical results and discussions}

\subsection{Validation}

We consider the Poiseuille flow in long rectangular channel with length $L_{1}$, height $L_{2}$ and width $L_{3}$. Without loss of generality, we can assume $L_{2} \leq L_{3}$. In addition, we consider the 
channel length is substantially larger than its width $\left(L_{1} \gg L_{3}\right)$ so the end effects can be neglected. In practice, the smallest dimension of the channel is taken as the characteristic length $\left(L=L_{2}\right)$. The reduced mass flow rate $G$, which normalized by $L_{2}^{2} L_{3} \Delta \hat{p} / v_{m} L_{1}$, is related to the dimensionless mass flow rate $G_{p}$ by $G=G_{p} L_{1} / L_{2}$.

When the aspect ratio $L_{2} / L_{3}$ approaches 0 , the rectangular channel flow reduces to plane channel flow, i.e., flow between two parallel plates. Simulations of plane channel flow are performed with DVM D2Q1600, LBM D2Q9 and computational domain of $145 \times 7$ grid points.

Figure 1.a) show the the perfect agreement in reduced mass flow rate $G$ obtained by the DVM $D 2 Q 1600$ with one from the linearised BGK model by variational method [39] for the plane channel flow $\left(L_{2} / L_{3}=0\right)$. The LBM D2Q9 gives fairly good agreement with the two mentioned approaches until $K n=0.1$ and considerably over-predicts $G$ at larger Knudsen numbers. In this Figure, the numerical solution from the linearised BGK model [40] and the experimental data [41] for the rectangular channel with small aspect ratio $L_{2} / L_{3}=0.02,0.019$, respectively, are also presented. For the flow in the plane channel $\left(L_{2} / L_{3}=0\right)$ and the rectangular channel $\left(L_{2} / L_{3}=0.02\right)$, the lateral walls have almost no effect in the slip and transitional flow regimes, but significantly reduce $G$ in the free molecular flow regime $(K n>10)$. Our DVM $D 2 Q 1600$ data agree well with measurements until the early free molecular flow regime. In the full free molecular flow regime $(K n>40)$, the measurements are closer to the numerical results for the rectangular channel than our numerical results for the plane channel. This may be attributed to the lateral wall effect in the rectangular channel, which becomes significant as gas molecules are more likely to collide with the lateral walls at high Knudsen numbers. It is noted that, in this Figure, all the numerical data are obtained with diffuse boundary condition on the channel walls $\left(\alpha_{t}=1\right)$. 


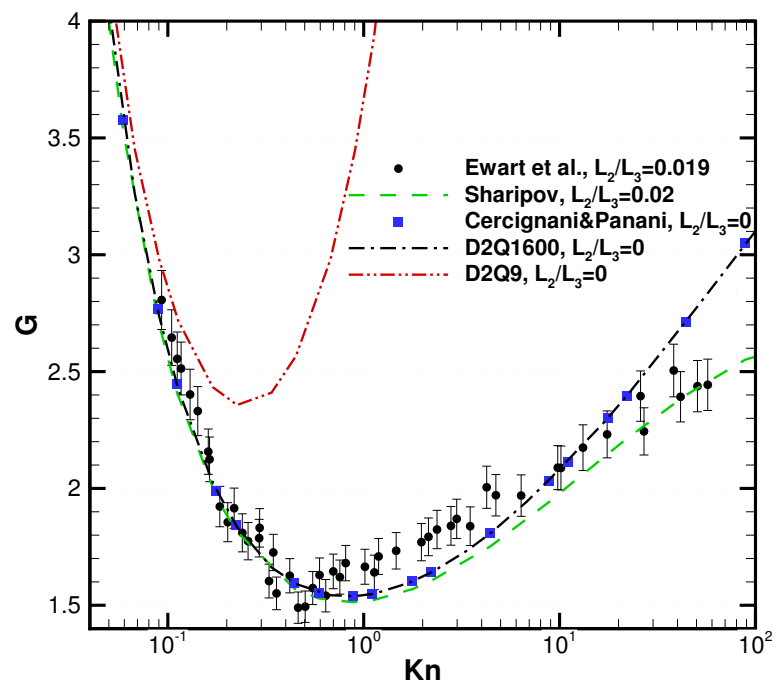

(a) Diffusive boundary condition

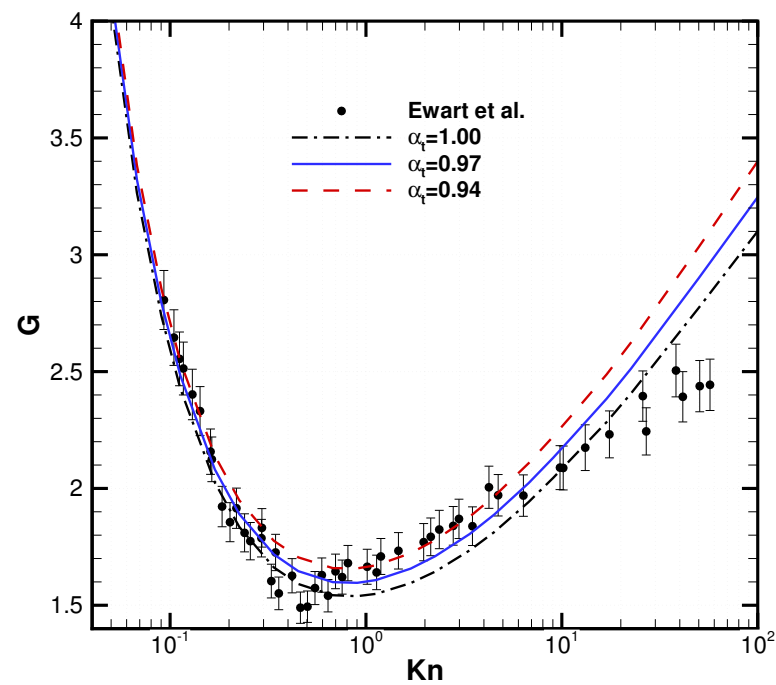

(b) Diffusive-specular boundary condition

Figure 1: Comparison of reduced mass flow rate $G$ in a plane channel $\left(L_{2} / L_{3}=0\right)$ with the other numerical data for a plane channel $\left(L_{2} / L_{3}=0\right)$ [39] and a rectangular channel $\left(L_{2} / L_{3}=0.02\right)$ respectively [40], and the experimental data for a rectangular channel $\left(L_{2} / L_{3}=0.019\right)$ [41]. All the numerical data are obtained by solving linearised BGK equation. 


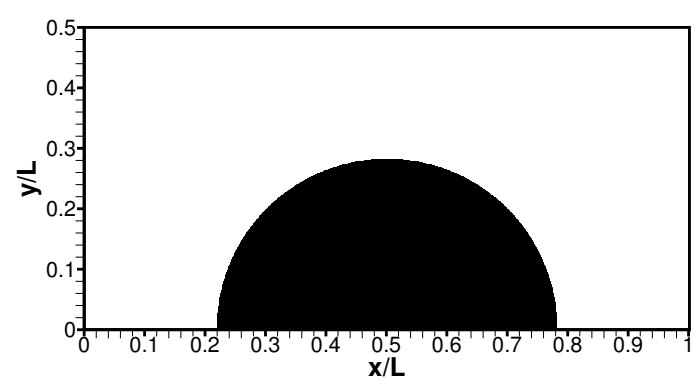

(a) Square array of circular cylinder

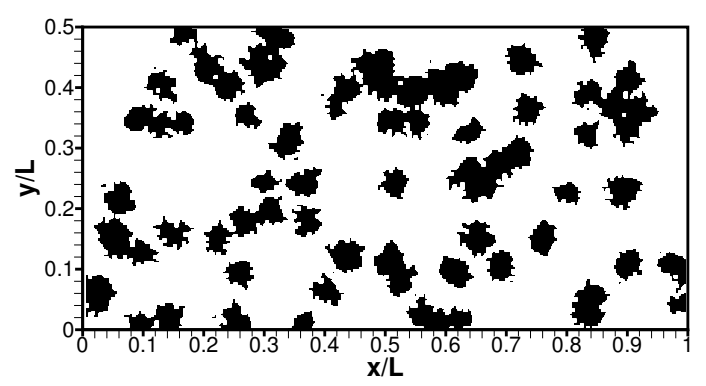

(b) Random porous media generated by QSGS

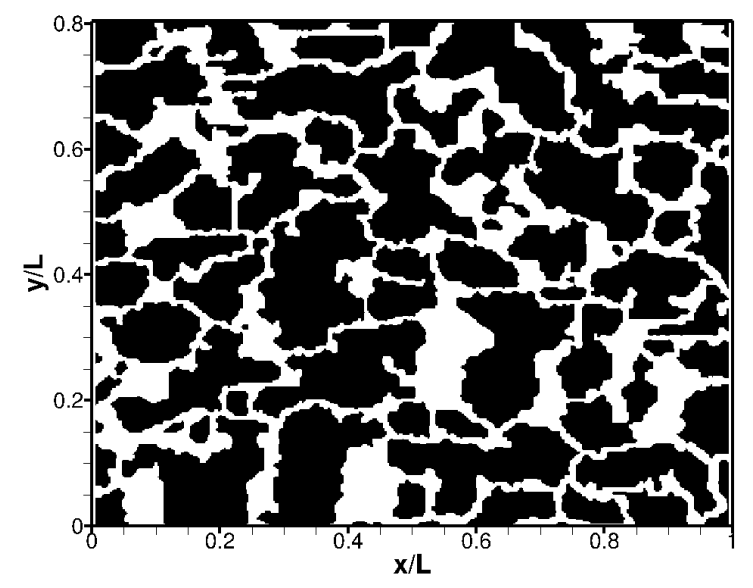

(c) 2D micromodel of Berea sandstone

Figure 2: 2D digital models of porous media, in which the black and white regions represent the matrix and void, respectively. The image resolution $N_{x}$ and porosity $\epsilon$ are (a) $N_{x}=800 \times 400, \epsilon=0.75$; (b) $N_{x}=800 \times 400, \epsilon=0.75$; and (c) $N_{x}=1597 \times 1282, \epsilon=0.32$. 
randomly generated by the quartet structure generation set (QSGS) [42, 43], and the micromodel of Berea sandstone [44] as shown in Fig. 2. The first two 2D models are artificially generated with the same porosity $\epsilon$ of 0.75 , while the third model with $\epsilon=0.32$ is extracted from a realistic rock sample. We simulate the flows in these porous media covering all the flow regimes with $K n$ ranging from $10^{-4}$ to 10 . In the DVM simulations, different resolutions in the velocity space, i.e. $D 2 Q 16, D 2 Q 36, D 2 Q 64, D 2 Q 1600$ are used, while the $\mathrm{D} 2 \mathrm{Q} 9$ model is employed for the LBM simulations. The intrinsic permeability $k_{\infty}$ at $K n=0$ is extrapolated from the apparent permeability $k$ obtained by the LBM simulations with the bounce-back boundary condition in the hydrodynamic flow regime. The accuracy of our DVM solver has been validated in our previous work [45].

Apparent permeability $k$ obtained by the DVM simulations using a refined molecular velocity grid $D 2 Q 1600$ (or $D 2 Q 64$ which yields the almost identical data as the $D 2 Q 1600$ at sufficiently small $K n$ ) is tabulated in Table 1. For any $K n$, the apparent permeability decreases considerably from the cylinder model, the QSGS model, to the Berea stone model, as the flow geometry becomes more complicated.

Table 1: The (dimensionless) apparent permeability $k$ obtained by the DVM simulations for three 2D geometries at different $K n$

\begin{tabular}{lrrr}
\hline Geometry in Fig. 2 & (a) Cylinder & (b) QSGS & (c) Berea \\
\cline { 3 - 4 }$K n$ & $K n_{l} / K n=2.137$ & $K n_{l} / K n=29.01$ & $K n_{l} / K n=538.8$ \\
\cline { 2 - 4 } $10^{-4}$ & $1.355 \times 10^{-2}$ & $6.887 \times 10^{-5}$ & $1.411 \times 10^{-1}$ \\
$10^{-3}$ & $1.385 \times 10^{-2}$ & $9.093 \times 10^{-5}$ & $3.329 \times 10^{-7}$ \\
$10^{-2}$ & $1.533 \times 10^{-2}$ & $2.025 \times 10^{-4}$ & $2.107 \times 10^{-6}$ \\
$10^{-1}$ & $3.120 \times 10^{-2}$ & $1.266 \times 10^{-3}$ & $1.953 \times 10^{-5}$ \\
$10^{0}$ & $2.189 \times 10^{-1}$ & $1.177 \times 10^{-2}$ & $1.960 \times 10^{-4}$ \\
$10^{1}$ & $3.701 \times 10^{0}$ & $1.165 \times 10^{-1}$ & $1.958 \times 10^{-3}$ \\
\hline
\end{tabular}

We now consider the permeability correction $k / k_{\infty}$ with respect to the level of rarefaction. In 


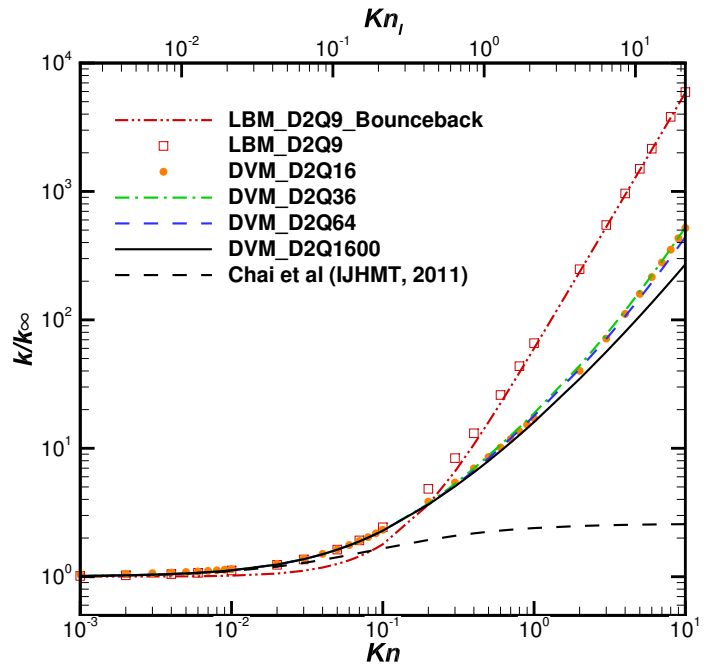

(a) Square array of circular cylinder

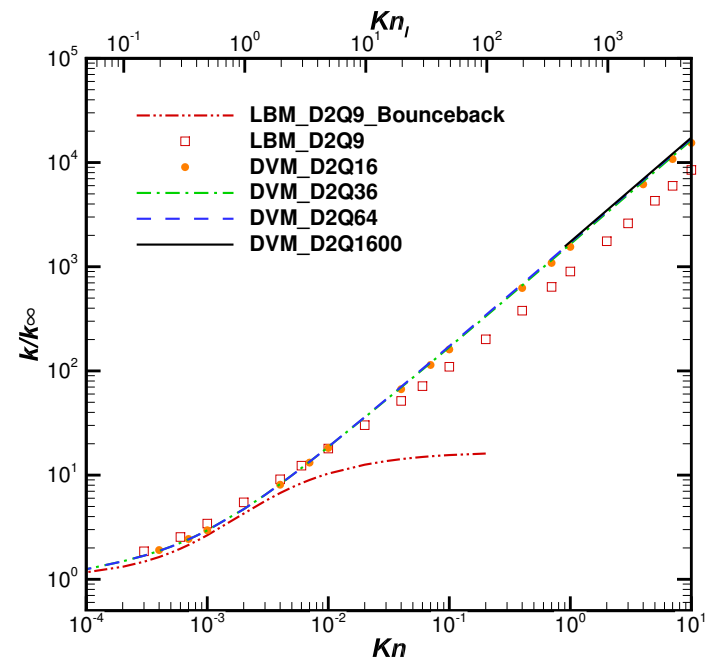

(c) Berea sandstone model

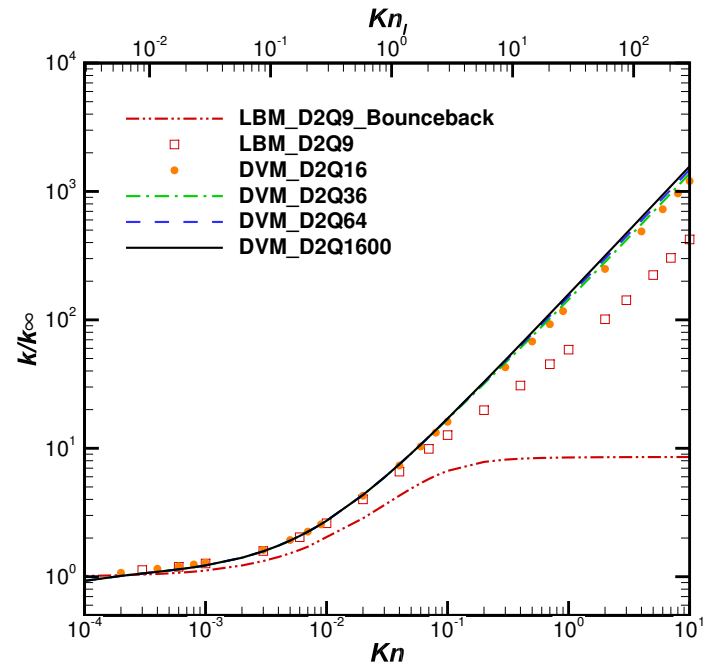

(b) QSGS model

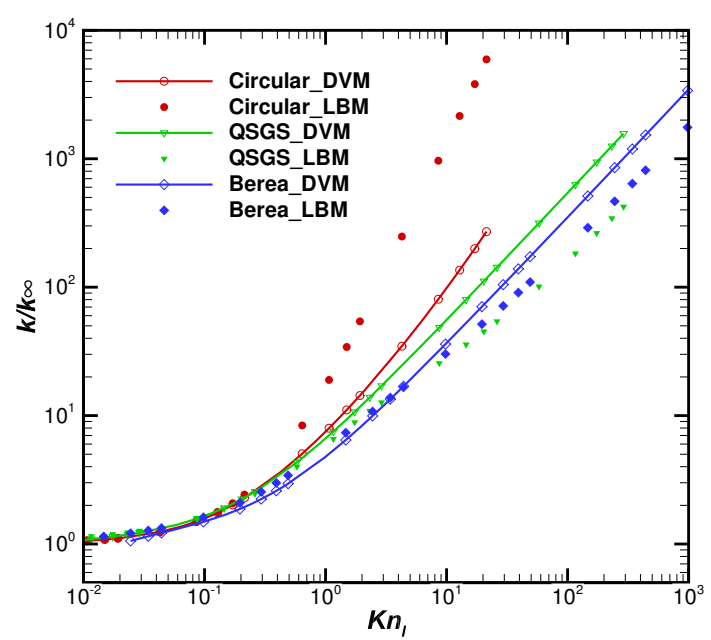

(d) Plot with effective Knudsen number $K n_{l}$

Figure 3: Permeability correction $k / k_{\infty}$ versus Knudsen number $K n$ obtained by the LBM and DVM simulations for the $2 \mathrm{D}$ porous models. 
the first case, the unit cell is a square with a circle positioned at its centre and repeated itself in the 2D space. The computational domain of $400 \times 800$ grid points contains only half of the unit cell as shown in Fig. 2(a) thanks to the symmetry and periodicity of the configuration. The effective Knudsen number $K n_{l}=2.137 K n$ is estimated with $k_{\infty}=1.368 \times 10^{-2}$. Figure 3 (a) demonstrates consistency of the permeability correction $k / k_{\infty}$ obtained by all the examined approaches in the continuum limit $\left(K n_{l} \lesssim 0.005\right)$. However, in the slip regime, the effect of boundary condition on the LBM simulation can be observed: the LBM data with the bounce-back and diffuse boundary conditions start to deviate from the DVM results at $K n_{l} \approx 0.01$ and $K n_{l} \approx 0.2$, respectively. Because the diffuse boundary condition can capture velocity slip while the bounce-back scheme produces non-slip boundary condition, it is not surprising that the LBM with diffuse boundary condition perform better in the slip regime [46, 47]. The DVM data show that the analytic solution derived from the Stokes approximation with the Maxwell's slip boundary condition [48] is valid when $K n_{l} \lesssim 0.05$. The effect of resolution in the molecular velocity space is pronounced in the DVM simulations when $K n_{l} \gtrsim 3$ and the largest discrepancy between the $D 2 Q 16$ and $D 2 Q 1600$ models is about $192 \%$ at $K n_{l} \approx 20$.

By retaining the same porosity $\epsilon=0.75$ of the cylindrical configuration, we examine a more complex geometry, i.e. randomly-generated porous geometry using the QSGS, as shown in Fig. 2(b). The effective Knudsen number $K n_{l}=29.01 K n$ is estimated with $k_{\infty}=6.317 \times 10^{-5}$. From Fig. 3(b), the permeability correction predicted by the LBM and DVM simulations with the diffuse boundary condition are in good agreement when $K n_{l} \lesssim 1$. When $K n_{l}$ increases further, they start to deviate rapidly. Compared to the cylindrical configuration, the largest difference in the permeability correction obtained by the DVM simulations with different velocity resolutions be- 
comes significantly smaller, e.g. the difference of $24 \%$ for the highly rarefied flow with $K n_{l} \approx 200$. Meanwhile, the DVM simulation will suffer large numerical diffusion in the hydrodynamic regime which is caused by insufficient spatial resolution, so the permeability correction is slightly smaller than unity at $K n=10^{-4}$. As the LBM was originally developed for the hydrodynamic flows [17], its streaming and collision scheme can adsorb numerical diffusion using the effective relaxation time of $\tilde{\tau}=\tau+0.5$. In general, DVM requires a finer spatial grid in the hydrodynamic flow regime than the LBM [49].

With porosity $\epsilon$ of 0.32 , the 2D micromodel of Berea sandstone, see Fig. 2(c), represents a more complex and realistic porous medium with highly tortuous flow paths. The effective Knudsen number $K n_{l}=538.8 K n$ is calculated with $k_{\infty}=1.129 \times 10^{-7}$. With the reference length $L=1774 \mu m$, the dimensional intrinsic permeability is $\hat{k}_{\infty}=k_{\infty} \times L^{2}=360 m D$, while the experimental value of $445 \pm 35 \mathrm{mD}$ is measured from the thin section of the 3D Berea sandstone rock sample [44]. Figure 3(c) indicates that the resolution of velocity grid becomes less important in all the flow regimes with this complex sandstone sample. The maximum difference of the permeability correction predicted by the $D 2 Q 16$ and $D 2 Q 1600$ models diminishes to approximately $11 \%$ at $K n_{l} \approx 4000$. As the large flow paths with least resistance dominate the overall permeability in this sandstone sample, the low resolution $D 2 Q 16$ model is sufficient to describe less rarefied flows accurately in these large flow paths.

Figure 3(d) plots the permeability correction in these three cases at different effective Knudsen number $K n_{l}$. Only the accurate high-resolution data obtained from the DVM (D2Q1600) simulations are retained, which are compared with the results of the LBM D2Q9 model using the diffuse boundary condition. Although the three examined geometries are completely different, 
dependency of the permeability correction on the effective Knudsen number in the slip and early transition flow regimes are almost identical. This demonstrates that the effective Knudsen number $K n_{l}$ can reasonably characterise the rarefaction effects in these porous media. Considering the flow in the early free molecular regime, i.e. $K n_{l} \approx 20$, the relative difference between the LBM and DVM results reduces with complexity of the porous geometries, which is roughly $2096 \%$, $-60 \%$ and $-27 \%$ for the cylindrical, random and sandstone geometries, respectively, suggesting that the overall permeability is mainly determined by the less rarefied flow in large flow paths where the LBM considering velocity-slip may be sufficiently accurate.

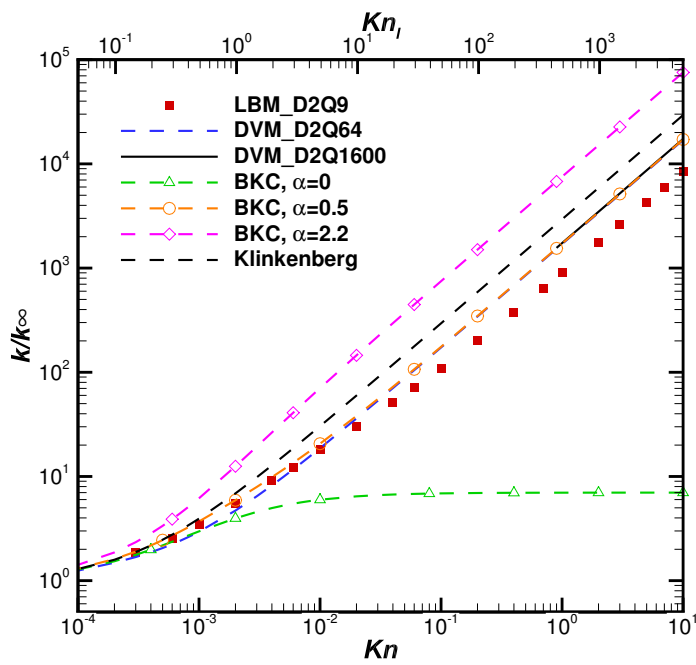

Figure 4: Permeability correction $k / k_{\infty}$ versus Knudsen number obtained by the LBM and DVM simulations, and the Klinkenberg and $\mathrm{BKC}$ models for the micromodel of Berea sandstone.

The Klinkenberg-type models are analysed for the micromodel of Berea sandstone. Figure 4 compares the permeability correction predicted by the Klinkenberg and BKC models, see Eq. (25) and Eq. (26). The BKC model with the empirical parameter $\alpha=0$, i.e. the viscosity is assumed to be constant in all the flow regimes, underestimates the permeability correction in the transition 
and free-molecular regimes. However, the BKC model with $\alpha=2.2$, i.e. second-order correction for a $2 \mathrm{D}$ channel of length-to-height ratio of 20 , overestimates the permeability correction in the slip regime. It is found that the BKC model with $\alpha=0.5$ performs well in comparison with the accurate DVM (D2Q1600) data. Note: the $D 2 Q 1600$ data are almost identical to the $D 2 Q 64$ results, so they are only presented for $K n>1$ in Figure 4. Meanwhile, the Klinkenberg model overestimates the permeability correction in the transition and free-molecular regimes by $70 \%$.

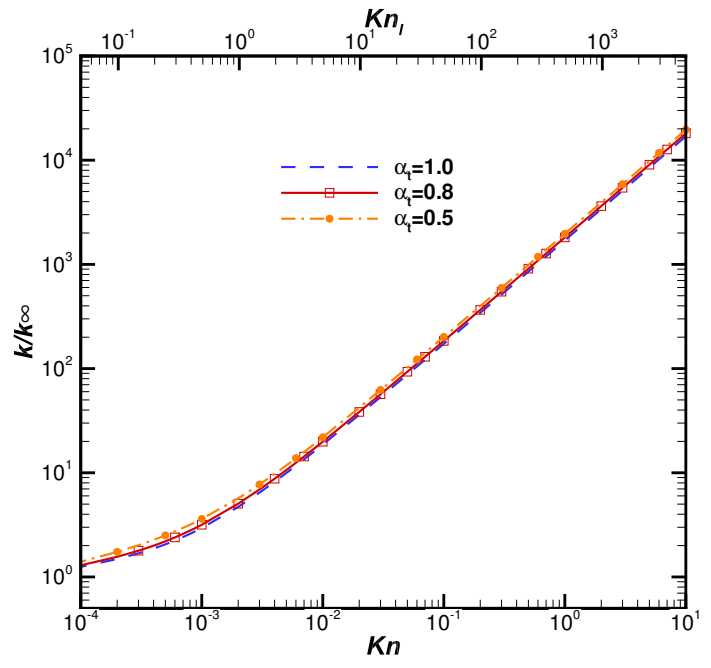

Figure 5: Permeability correction $k / k_{\infty}$ versus Knudsen number obtained by the DVM D2Q64 with different TMAC for the micromodel of Berea sandstone.

The influence of TMAC $\alpha_{t}$ on permeability correction for the micromodel of Berea sandstone is illustrated by DVM D2Q64 data with $\alpha_{t}=1.0,0.8,0.5$ in Figure 5. Similar to the case of channel flow shown in Figure 1 , decreasing $\alpha_{t}$ slightly increases the permeability correction $k / k_{\infty}$. Permeability correction dependence on TMAC for array of square cylinders has been analysed in our previous work [45]. From that study, the permeability correction also increases with reducing $\alpha_{t}$. The accuracy of slip-corrected model, e.g. the Klinkenberg correction, becomes considerably 


\subsection{D porous media}

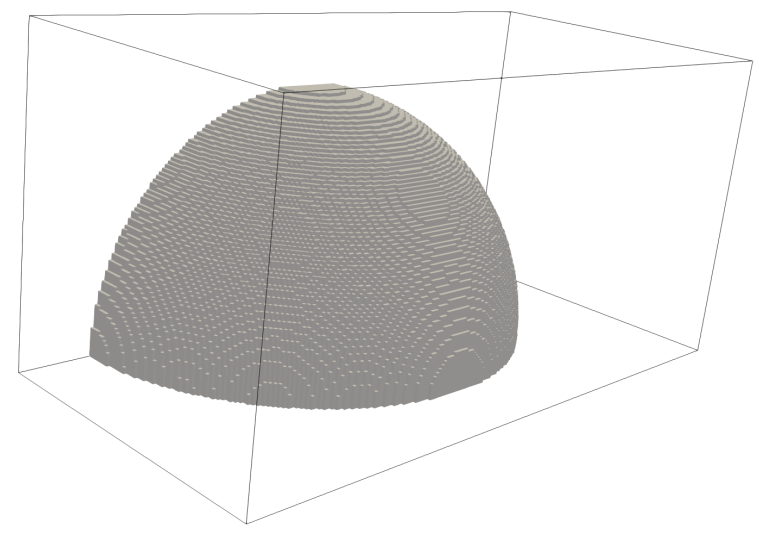

(a) Cubic sphere packing

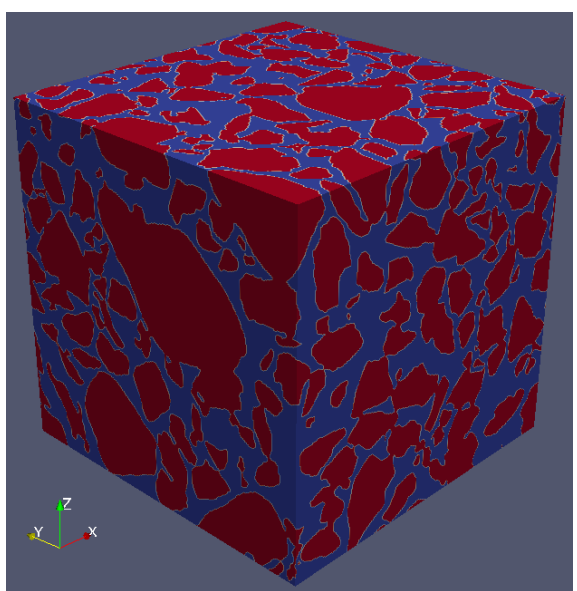

(b) Sand-pack sample

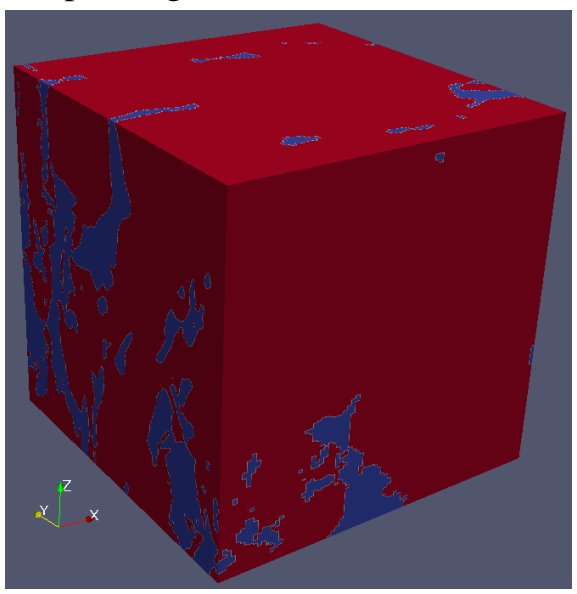

(c) Shale sample

Figure 6: 3D digital models of porous media, in which the matrix is represented by (a) grey colour; (b) \& (c) red colour. The image resolution $N_{x}$ and porosity $\epsilon$ are (a) $N_{x}=400 \times 200 \times 200, \epsilon=0.75$; (b) $N_{x}=300 \times 300 \times 300$, $\epsilon=0.38 ;$ and (c) $N_{x}=400 \times 400 \times 400, \epsilon=0.17$.

We now investigate rarefied flows in 3D porous media including a simple cubic array of spheres, a sand-pack sample, and a shale rock sample, see Fig. 6. In the first case, the sphere model is artificially generated with the porosity $\epsilon$ of 0.75 , while for the sand-pack mode ${ }^{1}, \epsilon=0.38$,

\footnotetext{
${ }^{1}$ Retrieved from http://xct.anu.edu.au/network_comparison/\#data_sets
} 
$L=2.754 \mathrm{~mm}$, and the shale model is reconstructed from the Fayetteville shale sample [50], which has $\epsilon=0.17$ and $L=4.6 \mu \mathrm{m}$. In the first case, the unit cell is a cubic box with a sphere placed at its centre and repeated itself in the 3D space. The computational domain contains only a quarter of the unit cell as shown in Fig. 6(a) because of the symmetry and periodicity of the configuration. To ensure a wide range of rarefaction is considered, we set up our simulations with $K n=10^{-3}, 10^{-2}, 10^{-1}, 1$ for each geometry. The intrinsic permeability is obtained by the LBM simulations using the D3Q19 model with the bounce-back scheme on solid surfaces at $K n=10^{-4}$, and different resolution in the molecular velocity space, i.e. $D 3 Q 64, D 3 Q 216$, $D 3 Q 1728$, are considered in the DVM simulations. The intrinsic permeability $k_{\infty}$ is calculated to be $1.490 \times 10^{-2}, 9.819 \times 10^{-6}$, and $8.074 \times 10^{-6}$ for the sphere and sand-pack models and the rock sample, respectively. The value of $k_{\infty}=1.497 \times 10^{-2}$ was reported for the sphere model by solving the Stokes equations [51], which is very close to our computed value. The intrinsic permeability of sand-pack model is only $21.6 \%$ larger than that of shale model while the porosity is significantly bigger, leading to smaller (dimensionless) average pore size $L^{*} / L=K n / K n_{l}$, see Eq. (24).

The apparent permeability $k$ obtained by the DVM simulations with the finest molecular velocity grid at different levels of rarefaction is listed in Table 2. The apparent permeability at a specific $K n$ is found to decrease when the porous flow geometry becomes more complex, which is consistent with the finding from the 2D cases. Although the (dimensionless) apparent permeability $k$ of sand-pack and shale samples are in the same order of magnitude, the characteristic length $L$, i.e. rock size, of shale is smaller than that of sand-pack by three-order of magnitude. Therefore, the dimensional apparent permeability $\hat{k}$ of shale sample is six-order of magnitude smaller than 
that of sand-pack sample at the same $K n$.

Table 2: The (dimensionless) apparent permeability $k$ obtained by the DVM simulations. The dimensional apparent permeability can be obtained by $\hat{k}=k L^{2}$, where the rock sizes of sand-pack and shale samples are $L=2.754 \times$ $10^{-3} \mathrm{~m}, 4.6 \times 10^{-6} \mathrm{~m}$ respectively.

\begin{tabular}{lrrr}
\hline Geometry in Fig. 6 & $\begin{array}{r}\text { (a) Sphere } \\
\text { (b) Sand-pack }\end{array}$ & $\begin{array}{r}\text { (c) Shale } \\
K n_{l} / K n=2.506\end{array}$ \\
\cline { 1 - 3 } $1 n_{l} / K n=69.55$ & $K n_{l} / K n=51.30$ \\
$10^{-3}$ & $1.515 \times 10^{-2}$ & $1.251 \times 10^{-5}$ & $1.000 \times 10^{-5}$ \\
$10^{-2}$ & $1.730 \times 10^{-2}$ & $4.272 \times 10^{-5}$ & $2.454 \times 10^{-5}$ \\
$10^{-1}$ & $3.871 \times 10^{-2}$ & $3.392 \times 10^{-4}$ & $1.536 \times 10^{-4}$ \\
$10^{0}$ & $2.237 \times 10^{-1}$ & $3.324 \times 10^{-3}$ & $1.367 \times 10^{-3}$ \\
\hline
\end{tabular}

The permeability correction for the 3D porous models is also found to increase with $K n$ which is consistent with the 2D cases, see Fig. 7. Again, the LBM with the bounce-back scheme cannot predict accurate permeability correction in the slip flow regime while the kinetic boundary condition, which can help to capture velocity-slip at solid surface, can extend the validity of the LBM D3Q19 model to the early transition flow regime. While the permeability correction increases with $K n$, the resolution of the velocity space becomes less important for accurate DVM simulations with increasing complexity of porous geometries. For example, for highly rarefied flow with $K n=1$, the predicted permeability by the $D 3 Q 64$ model deviates $5.7 \%$ from the more accurate D3Q216 model for the simple sphere model, while small difference of $0.6 \%$ is observed between the $D 3 Q 64$ model and the more accurate $D 3 Q 1728$ model for the sand-pack porous medium. This is because the overall permeability is mainly determined by the less rarefied flow in large flow paths where the low-resolution DVM is sufficiently accurate. This finding has significant indication to pore-scale direct simulation for the real rock samples. To provide accurate prediction of the permeability which is a global parameter, DVM simulation with relatively low resolution i.e. D3Q64 is sufficient to capture rarefaction effects, resulting in significant reduction of com- 


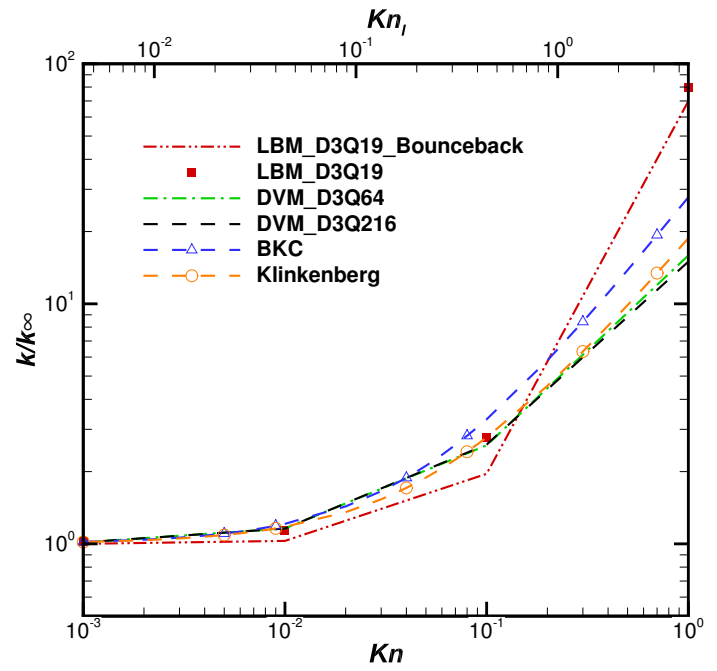

(a) Cubic sphere packing

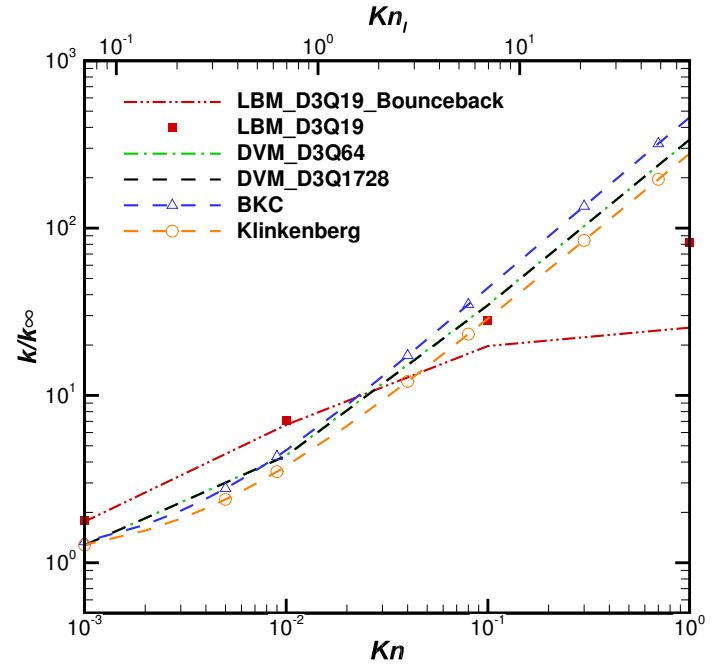

(b) Sand-pack sample

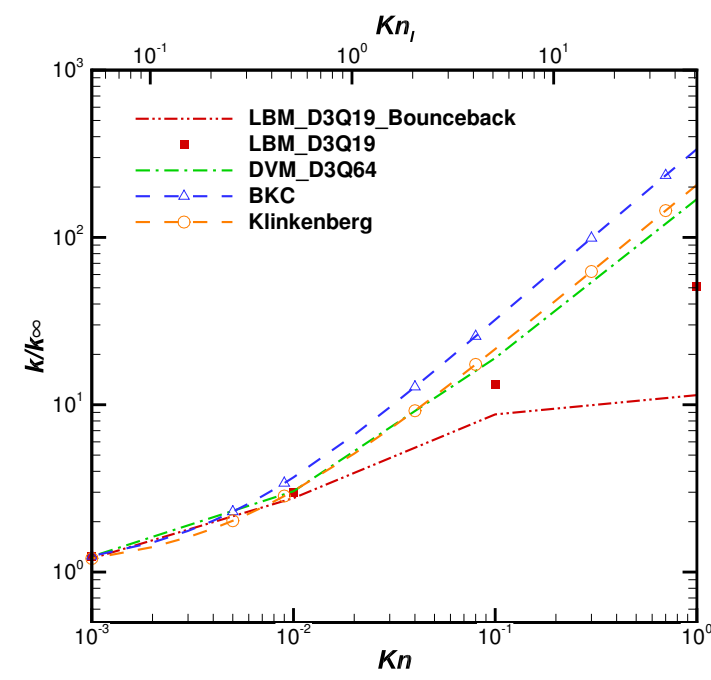

(c) Shale sample

Figure 7: Permeability correction $k / k_{\infty}$ versus Knudsen number $K n$ for the 3D porous models. 
putational cost. Meanwhile, the LBM cannot capture rarefaction effect. For example, for highly rarefied flow, the LBM over-predicts permeability correction for the case of cubic array of spheres but under-predicts for the other cases.

It is also demonstrated that the BKC model always yields higher permeability correction than the Klinkenberg model. The difference between them increases noticeably with the rarefaction level which grows to around $55 \%$ at $K n_{l}=10$. For highly rarefied flows, the DVM are lower than these two heuristic models for the sphere and shale cases but are between them for the sandpack case. To use these heuristic Klinkenberg-type models, empirical parameters, such as $c, \tau_{h}$ in Eqs. [25], (28) need to be calibrated for each porous medium [21, 20]. For more sophisticated model, e.g. the APF of Eq. 29), additional parameters $\delta^{\prime}$ and $D_{f}$ need to be evaluated. As these empirical parameters are different for each sample and also depend on flow conditions, these Klinkenberg-type models cannot be of any practical use for ultra-tight porous media.

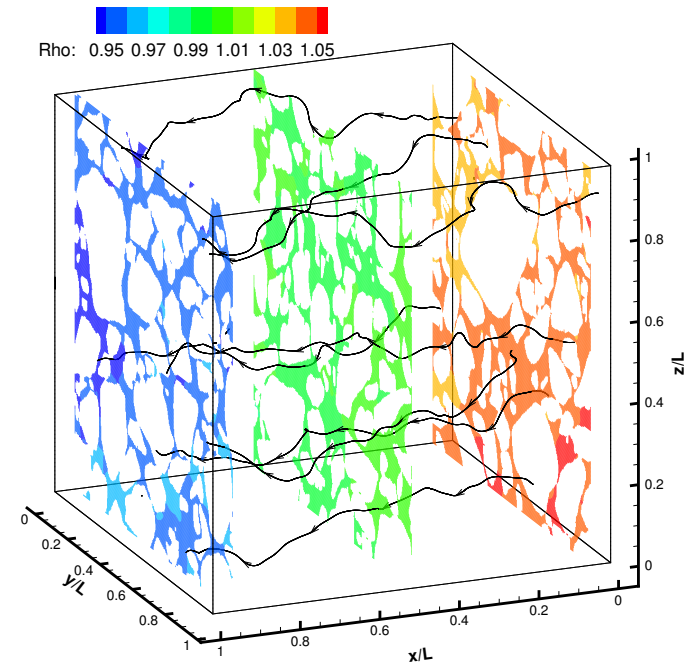

(a) Sand-pack sample

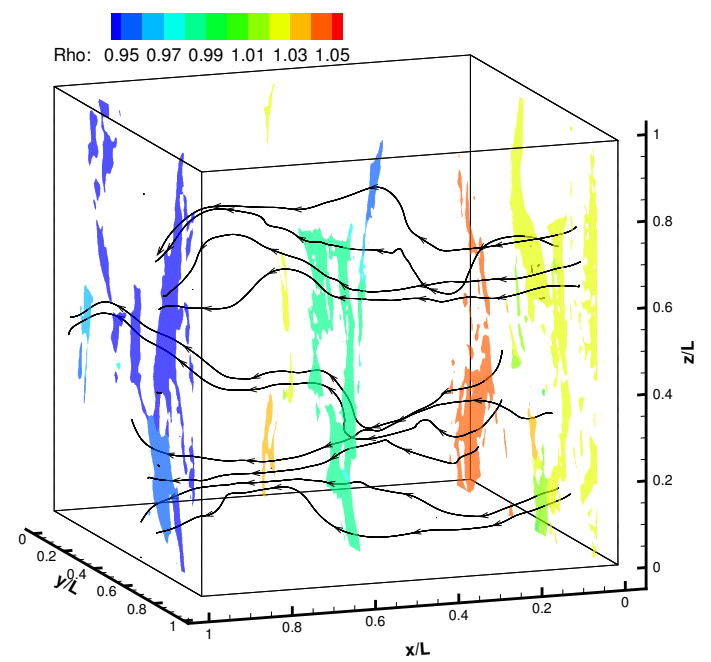

(b) Shale sample

Figure 8: Streamlines (arrows) and distribution of density (Rho) at the three cross sections $\hat{x} / L=0.05,0.5,0.95$. 


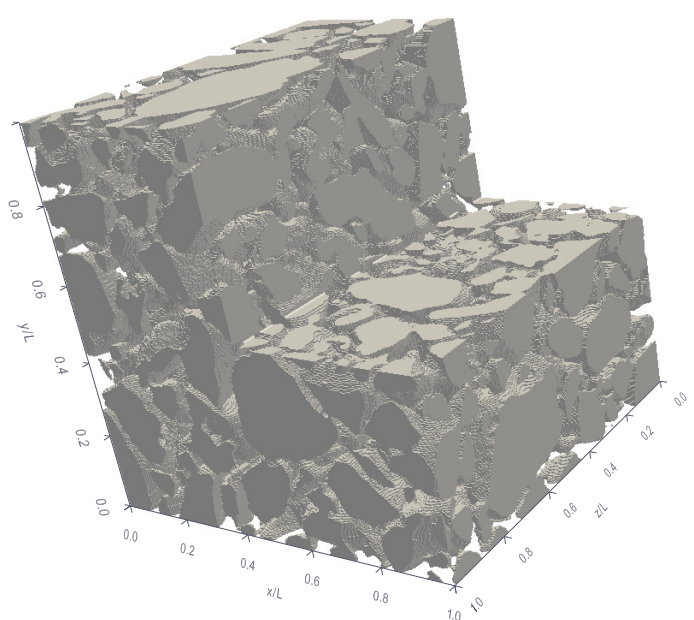

(a) Sand-pack sample

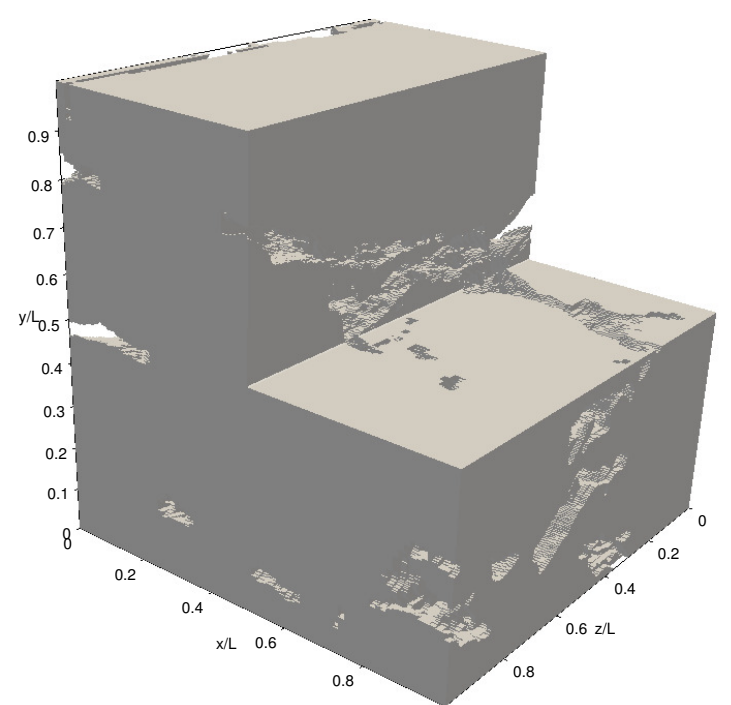

(b) Shale sample

Figure 9: Cut-off view of the samples

Figure 8 depicts the streamlines and distribution of density at three cross sections $\hat{x} / L=$ 0.05, 0.5, 0.95 obtained by DVM for the sand-pack and shale rock samples. Highly tortuous streamlines in both porous media can be observed. However, the density in each stream-wise cross section of sand-pack model is more homogeneous than that of shale model, which may be due to more cross-linked pores (in y-z plane) in the sand-pack sample while there are more isolated pores in the shale sample. It can also be seen from Fig. 9, there are more throats or pore constrictions in the sand-pack model than the shale sample, which leads to larger flow resistance in the sand-pack model. Therefore, these samples have similar intrinsic permeability although their porosities are very different. The combination of relative big pores with narrow throats in the sand-pack sample may result in smaller (dimensionless) average pore size than the shale sample.

To assess the practical use of the two numerical methods, we compare the computational cost of LBM D2Q9 and DVM D2Q16 for the 2D Berea sample; LBM D3Q19 and DVM D3Q64 for 
the 3D shale sample at $K n=10^{-1}$. Wall clock time of serial calculation on a single core of Intel i74770 processor of a workstation (Dell Precision Tower 7910) is recorded for fair comparison. The wall clock times of the LBM and DVM simulations are 5.65 and 1.26 hours respectively for the 2D Berea sample, and are 40.29 and 649.3 hours respectively for the 3D shale rock sample. The difference is mainly owning to the number of discrete velocity, approximation of advection term and convergence rate. We have recently developed a multi-level parallel DVM solver to enable practical pore-scale simulation, where the parallel performance of the solver is analyzed [52].

\section{Conclusions and remarks}

Here, we have used the gas kinetic model to directly simulate gas flows inside the porous media utilising the digital images of porous media to accurately predict the permeability enhancement due to rarefaction effects. The commonly-used standard LBM cannot capture rarefaction effects, although the kinetic boundary condition, which captures velocity-slip at surface, can extend the validity of the LBM to the slip flow regime. While the Klinkenberg model extends the validity of the Darcy law to the slip flow regime, the heuristically extended Klinkenberg-type models to all the flow regime, e.g. the $\mathrm{BKC}$ model, are not of any practical use because of many unknown empirical parameters which need to be calibrated for each porous media. And even worse, these parameters also depend on flow conditions. By contrast, our kinetic solver can accurately predict apparent permeability without introducing any empirical parameters, which offers a promising new way for digital rock analysis. As flows in the large flow paths are less rarefied, which have dominant contribution to the overall permeability, the requirement on the velocity-space resolution can be significantly reduced for the DVM simulations, resulting in more affordable computational 
costs.

\section{Acknowledgements}

Financial support from the UK Engineering and Physical Sciences Research Council (EPSRC) under grant no. EP/M021475/1 is gratefully acknowledged. L. Zhu acknowledges the financial support from the Chinese Scholarship Council (CSC) during his visit to the UK (CSC Student no. 201606160050). L. Wu acknowledges the financial support from the RSE-NSFC joint project and Carnegie Research Incentive Grant. Results were obtained using the EPSRC funded ARCHIEWeSt High Performance Computer (EPSRC Grant No. EP/K000586/1) and the ARCHER national supercomputer through the UK Consortium on Mesoscale Engineering Sciences (EPSRC Grants No. EP/L00030X/1 and EP/R029598/1). The authors would like to thank Prof. Baojun Bai for providing access to the digital shale image.

\section{References}

[1] C. McGlade, J. Speirs, S. Sorrell, Unconventional gas : a review of regional and global resource estimates, Energy 55 (2013) 571-584. doi : 10.1016/j .energy .2013.01.048

[2] Golden rules for a golden age of gas, special report on unconventional gas, Tech. rep., International Energy Administration, Paris (2012).

[3] P. J. M. Monteiro, C. H. Rycroft, G. I. Barenblatt, A mathematical model of fluid and gas flow in nanoporous media, Proc Natl Acad Sci 109 (50) (2012) 20309-20313. doi:10.1073/pnas.1219009109.

[4] T. W. Patzek, F. Male, M. Marder, Gas production in the Barnett Shale obeys a simple scaling theory, Proc Natl Acad Sci 110 (49) (2013) 19731-19736. arXiv:arXiv: 1408.1149, doi: 10.1073/pnas.1313380110.

[5] J. C. Maxwell, On stresses in rarified gases arising from inequalities of temperature, Philos Trans R Soc London 170 (1879) 231-256. doi:10.1098/rstl.1879.0067.

[6] F. Sharipov, Data on the velocity slip and temperature jump on a gas-solid interface, J Phys Chem Ref Data 40 (2) (2011) 023101. doi: 10.1063/1.3580290

[7] D. A. Lockerby, J. M. Reese, On the modelling of isothermal gas flows at the microscale, J Fluid Mech 604 (2008) 235-261. doi:10.1017/S0022112008001158

[8] L. Klinkenberg, The permeability of porous media to liquids and gases, Am Pet Inst (1941) API-41-200.

[9] F. A. Florence, J. A. Rushing, K. E. Newsham, T. A. Blasingame, Improved permeability prediction relations for low permeability sands, SPE Rocky Mt Oil Gas Technol Symp SPE 107954 (2007) 18. doi:10.2118/ 107954-MS.

[10] F. Civan, Effective correlation of apparent gas permeability in tight porous media, Transport Porous Med 82 (2) (2010) 375-384. doi:10.1007/s11242-009-9432-z

[11] H. Darabi, A. Ettehad, F. Javadpour, K. Sepehrnoori, Gas flow in ultra-tight shale strata, J Fluid Mech 710 (2012) 641-658. doi: 10.1017/jfm.2012.424. 
[12] A. S. Ziarani, R. Aguilera, Knudsen's permeability correction for tight porous media, Transport Porous Med 91 (1) (2012) 239-260. doi:10.1007/s11242-011-9842-6.

[13] C. White, T. J. Scanlon, R. E. Brown, Permeability of ablative materials under rarefied gas conditions, J Spacecr Rockets 53 (1) (2016) 134-142. doi:10.2514/1.A33279

[14] P. Bhatnagar, E. Gross, M. Krook, A model for collision processes in gases. I. Small amplitude processes in charged and neutral one-component systems, Phys Rev 94 (3) (1954) 511-525. doi:10.1103/PhysRev. 94. 511 .

[15] L. H. Holway, New statistical models for kinetic theory: methods of construction, Phys Fluids 9 (9) (1966) 1658-1673. doi: 10.1063/1.1761920

[16] E. M. Shakhov, Generalization of the Krook kinetic relaxation equation, Fluid Dyn 3 (5) (1968) 95-96. doi: 10.1007/BF01029546

[17] Y. H. Qian, D. D'Humières, P. Lallemand, Lattice BGK models for Navier-Stokes equation, Europhys Lett 17 (6) (1992) 479. doi : 10.1209/0295-5075/17/6/001

[18] M. J. Blunt, B. Bijeljic, H. Dong, O. Gharbi, S. Iglauer, P. Mostaghimi, A. Paluszny, C. Pentland, Pore-scale imaging and modelling, Adv Water Resour 51 (2013) 197-216. doi:10.1016/j .advwatres.2012.03.003

[19] L. Chen, Q. Kang, R. Pawar, Y. L. He, W. Q. Tao, Pore-scale prediction of transport properties in reconstructed nanostructures of organic matter in shales, Fuel 158 (2015) 650-658. doi:10.1016/j.fuel.2015.06.022

[20] L. Chen, L. Zhang, Q. Kang, H. S. Viswanathan, J. Yao, W. Tao, Nanoscale simulation of shale transport properties using the lattice Boltzmann method: permeability and diffusivity, Sci Rep 5 (2015) 8089. doi: 10.1038/srep08089

[21] J. Zhao, J. Yao, M. Zhang, L. Zhang, Y. Yang, H. Sun, S. An, A. Li, Study of gas flow characteristics in tight porous media with a microscale Lattice Boltzmann Model, Sci Rep 6 (1) (2016) 32393. doi:10.1038/ srep32393.

[22] J. Wang, L. Chen, Q. Kang, S. S. Rahman, The lattice Boltzmann method for isothermal micro-gaseous flow and its application in shale gas flow: A review, Int J Heat Mass Transf 95 (2016) 94-108. doi:10.1016/j. ijheatmasstransfer.2015.12.009

[23] S. H. Kim, H. Pitsch, I. D. Boyd, Accuracy of higher-order lattice Boltzmann methods for microscale flows with finite Knudsen numbers, J Comput Phys 227 (19) (2008) 8655-8671. doi:10.1016/j.jcp.2008.06.012.

[24] J. Meng, Y. Zhang, Accuracy analysis of high-order lattice Boltzmann models for rarefied gas flows, J Comput Phys 230 (3) (2011) 835-849. arXiv: arXiv:0908.4520v2, doi:10.1016/j .jcp.2010.10.023

[25] J. Meng, Y. Zhang, Gauss-Hermite quadratures and accuracy of lattice Boltzmann models for nonequilibrium gas flows, Phys Rev E 83 (3) (2011) 036704. doi: 10.1103/PhysRevE. 83.036704

[26] J. E. Broadwell, Study of rarefied shear flow by the discrete velocity method, J Fluid Mech 19 (3) (1964) 401414. doi: $10.1017 /$ S0022112064000817.

[27] J. Yang, J. Huang, Rarefied flow computations using nonlinear model Boltzmann equations, J Comput Phys 120 (2) (1995) 323-339. doi : 10.1006/jcph. 1995.1168.

[28] F. Sharipov, G. Bertoldo, Rarefied gas flow through a long tube of variable radius, J Vac Sci Technol A 23 (3) (2005) 531-533. doi:10.1116/1.1897703

[29] I. Graur, F. Sharipov, Gas flow through an elliptical tube over the whole range of the gas rarefaction, Eur J Mech B/Fluids 27 (3) (2008) 335-345. doi : 10.1016/j . euromechflu.2007.07.003

[30] S. Varoutis, S. Naris, V. Hauer, C. Day, D. Valougeorgis, Computational and experimental study of gas flows through long channels of various cross sections in the whole range of the Knudsen number, J Vac Sci Technol A 27 (1) (2009) 89. doi: 10.1116/1.3043463.

[31] F. Sharipov, I. A. Graur, Rarefied gas flow through a zigzag channel, Vacuum 86 (11) (2012) 1778-1782. doi: $10.1016 / \mathrm{j}$. vacuum.2012.02.028

[32] I. Graur, M. T. Ho, Rarefied gas flow through a long rectangular channel of variable cross section, Vacuum 101 (2014) 328-332. doi:10.1016/j . vacuum.2013.07.047.

[33] F. Sharipov, V. Seleznev, Data on internal rarefied gas flows, J Phys Chem Ref Data 27 (3) (1998) 657-706. doi: $10.1063 / 1.556019$.

[34] S. Chen, G. D. Doolen, Lattice Boltzmann Method for fluid flows, Annu Rev Fluid Mech 30 (1) (1998) 329-364. doi:10.1146/annurev.fluid.30.1.329 
[35] W. Gautschi, Algorithm 726 - ORTHPOL: A package of routines for generating orthogonal polynomials and Gauss-type quadrature rules, ACM Trans Math Softw 20 (1) (1994) 21-62. arXiv:9307212, doi:10.1145/ 292395.292467

[36] C. Cercignani, Mathematical methods in kinetic theory, 2nd Edition, Springer Science \& Bussiness Media New York, 1990. doi: 10.1007/978-1-4899-7291-0.

[37] S. Ansumali, I. V. Karlin, Kinetic boundary conditions in the lattice Boltzmann method, Phys Rev E 66 (2) (2002) 026311. arXiv:0206033, doi:10.1103/PhysRevE.66.026311

[38] A. Beskok, G. E. Karniadakis, Report: A model for flows in channels, pipes and ducts at micro and nano scales, Microscale Thermophys Eng 3 (1) (1999) 43-77. doi : 10.1080/108939599199864

[39] C. Cercignani, C. D. Pagani, Variational approach to boundary-value problems in kinetic theory, Phys Fluids 9 (6) (1966) 1167-1173. doi : 10.1063/1.1761816.

[40] F. Sharipov, Rarefied gas flow through a long rectangular channel, J Vac Sci Technol A 17 (5) (1999) 3062-3066. doi: 10.1116/1.582006

[41] T. Ewart, P. Perrier, I. A. Graur, J. G. Meolans, Mass flow rate measurements in a microchannel, from hydrodynamic to near free molecular regimes, J Fluid Mech 584 (2007) 337. doi:10.1017/S0022112007006374.

[42] M. Wang, J. Wang, N. Pan, S. Chen, Mesoscopic predictions of the effective thermal conductivity for microscale random porous media, Phys Rev E 75 (3) (2007) 1-10. doi:10.1103/PhysRevE.75.036702

[43] L. Germanou, M. T. Ho, Y. Zhang, L. Wu, Intrinsic and apparent gas permeability of heterogeneous and anisotropic ultra-tight porous media, J Nat Gas Sci Eng 60 (2018) 271-283. doi:10.1016/j.jngse.2018. 10.003

[44] E. S. Boek, M. Venturoli, Lattice-Boltzmann studies of fluid flow in porous media with realistic rock geometries, Comput Math Appl 59 (7) (2010) 2305-2314. doi:10.1016/j . camwa.2009.08.063.

[45] L. Wu, M. T. Ho, L. Germanou, X.-J. Gu, C. Liu, K. Xu, Y. Zhang, On the apparent permeability of porous media in rarefied gas flows, J Fluid Mech 822 (2017) 398-417. doi: 10.1017/jfm.2017.300

[46] V. Sofonea, R. F. Sekerka, Boundary conditions for the upwind finite difference Lattice Boltzmann model: Evidence of slip velocity in micro-channel flow, J Comput Phys 207 (2) (2005) 639-659. doi:10.1016/j. jcp.2005.02.003

[47] J. Meng, Y. Zhang, Diffuse reflection boundary condition for high-order lattice Boltzmann models with streaming-collision mechanism, J Comput Phys 258 (2014) 601-612. doi:10.1016/j .jcp.2013.10.057

[48] Z. Chai, J. Lu, B. Shi, Z. Guo, Gas slippage effect on the permeability of circular cylinders in a square array, Int J Heat Mass Transf 54 (13-14) (2011) 3009-3014. doi : 10.1016/j .ijheatmasstransfer.2011.02.049.

[49] P. Wang, M. T. Ho, L. Wu, Z. Guo, Y. Zhang, A comparative study of discrete velocity methods for low-speed rarefied gas flows, Comput Fluids 161 (2018) 33-46. arXiv:1612.06590, doi:10.1016/j.compfluid. 2017.11 .006

[50] B. Bai, M. Elgmati, H. Zhang, M. Wei, Rock characterization of Fayetteville shale gas plays, Fuel 105 (2013) 645-652. doi:10.1016/j. fuel.2012.09.043.

[51] A. Sangani, A. Acrivos, Slow flow through a periodic array of spheres, Int J Multiph Flow 8 (4) (1982) 343-360. doi: 10.1016/0301-9322(82)90047-7.

[52] M. T. Ho, L. Zhu, L. Wu, P. Wang, Z. Guo, Z.-H. Li, Y. Zhang, A multi-level parallel solver for rarefied gas flows in porous media, Comput Phys Commun 234 (2019) 14-25. arXiv:1712.01132, doi:10.1016/j. cpc.2018.08.009. 